\title{
Variable jets from young stars
}

\author{
A. C. Raga ${ }^{1}$, J. Cantó $^{2}$, A. Esquivel ${ }^{1}$, A. Rodríguez-González ${ }^{1}$, \\ P. F. Velázquez ${ }^{1}$ \\ ${ }^{1}$ Instituto de Ciencias Nucleares, Universidad Nacional Autónoma de México, Ap. 70-543, \\ 04510 D. F., México \\ email: raga@nucleares.unam.mx \\ ${ }^{2}$ Instituto de Astronomía, Universidad Nacional Autónoma de México, Ap. 70-468, 04510 D. \\ F., México
}

\begin{abstract}
In this paper we discuss the fact that the observed "accelerations" (i. e., higher velocities at larger distances from the source) observed along some Herbig-Haro (HH) jets directly imply that the ejection velocity has to be time-dependent. Even though discussed in the early literature of the subject, this is an often forgotten fact.
\end{abstract}

Keywords. ISM: kinematics and dynamics - ISM: jets and outflows - ISM: Herbig-Haro objects - stars: winds, outflows

\section{Introduction}

There are several different possibilities for interpreting the knots found along $\mathrm{HH}$ jets. The two best studied ones are:

- the identification of knots with shock cells produced by Kelvin-Helmholtz instabilities at the outer boundary of the jet beam (see Micono et al. 1998),

- modelling the knots as "internal working surfaces" produced by an ejection velocity variability (see Raga et al. 1990).

The most straightforward evidence favouring (in some objects) the second of these models is the existence of jet/counterjet systems with good knot-to-knot symmetries in the two outflow lobes (examples of this are HH 111, see Gredel \& Reipurth 1994 and HH 34, see García López et al. 2008).

In this paper, we concentrate on a second kind of evidence favouring the "internal working surface" scenario: the presence of "accelerating segments" along some jet beams (good examples of this being again HH 111, see Raga et al. 2002 and HH 34, see Heathcote \& Reipurth 1992).

\section{Accelerations}

Let us assume that we have a steady, one-dimensional gasdynamic jet. In the absence of gravity and radiative losses, the flow velocity $u$ along the outflow axis then obeys Bernoulli's theorem, which we write in the form:

$$
u^{2}=u_{0}^{2}\left[1+\frac{1}{\gamma-1}\left(\frac{1}{M_{0}^{2}}-\frac{c_{s}^{2}}{u_{0}^{2}}\right)\right],
$$

where $c_{s}$ is the sound speed at the position in which the flow velocity is $u$, and $M_{0}=$ $u_{0} / c_{s, 0}$ is the Mach number, $u_{0}$ the flow velocity and $c_{s, 0}$ the sound speed at the injection point. For a monoatomic gas, the specific heat ratio would be $\gamma=5 / 3$.

¿From equation (2.1), we see that at the injection point (in which $c_{s}=c_{s, 0}$ ) we correctly obtain $u=u_{0}$. Now, if the flow expands laterally as it flows along the jet 
axis, the resulting adiabatic expansion leads to a drop in the sound speed, and to a corresponding increase in the flow velocity (see equation 2.1). The maximum possible velocity that can be attained is:

$$
u_{\max }=u_{0} \sqrt{1+\frac{1}{(\gamma-1) M_{0}^{2}}} .
$$

For a $\mathrm{HH}$ jet, observations indicate that the injection Mach number is in the $M_{0} \sim 10 \rightarrow$ 30 range. Therefore, we would expect to see only very small variations (of at most $\sim 1 \%$ ) in the flow velocity as one moves along the jet beam.

However, there are many observations of quite substantial (of the order of $100 \%$ of the injection velocity) drops and "accelerations" in the flow velocity along HH jets. The drops in velocity in principle could be the result of (steady or non-steady) shocks within the jet beam. For the accelerations there is no possible mechanism other than a time-variability in the injection velocity.

In the discussion above, we have only considered a gasdynamic, non-radiative flow in the absence of an external gravitational field. The presence of gravity (e. g., from the stellar source or from the cloud core in which it is embedded) or of radiative losses will reduce the possible acceleration derived from equation (2.1). The presence of a magnetic field within the jet beam would also provide only small possible accelerations, provided that the Alfvénic Mach number at the injection point is large.

Therefore, all of the observed "accelerations" along HH jets are direct evidence for the existence of a time-variability in the ejection velocity. The theory of jets from variable sources is therefore a required ingredient for modelling many of the observed $\mathrm{HH}$ jets, and might also be relevant for modelling other $\mathrm{HH}$ objects in which clear accelerations have not yet been observed.

\section{Conclusions}

In this paper, we have discussed the fact that the highly supersonic "accelerations" (i. e., larger flow velocities at increasing distances from the outflow source) observed in some $\mathrm{HH}$ jets directly imply that the ejection velocity has to be time-dependent. This is a result of the fact that a high (gasdynamic and Alfvénic) Mach number jet has very little thermal and/or magnetic energy to convert into extra kinetic energy (i. e., to produce an outwards increase of the jet velocity). Therefore, jets with observed "accelerations" are direct evidence for the existence of a variable ejection.

\section{Acknowledgements}

This work was supported by the CONACyT grants 61547 and 101356 .

\section{References}

García López, R., Nisini, B., Giannini, T., Eislöffel, J., Bacciotti, F., \& Podio, L. 1008, A\&A, 487,1019

Gredel, R. \& Reipurth, B. 1994, ApJ 407, L19

Heathcote, S. \& Reipurth, B. 1992, AJ, 104, 2193

Micono, M., Massaglia, S., Bodo, G., Rossi, P., \& Ferrari, A. 1998, A\&A 333, 1001

Raga, A. C., Noriega-Crespo, A., Reipurth, B., Garnavich, P. M., Heathcote, S., Böhm, K. H., \& Curiel, S. 2002, ApJ, 565, L29

Raga, A. C., Cantó, J., Binette, L., \& Calvet, N. 1990, ApJ, 364, 601 